\title{
Effects of Nanoparticles on Pin-Based Microchannel Heat Sinks
}

\author{
Mohammad Zargartalebi ${ }^{1}$, Jalel Azaiez ${ }^{1}$ \\ ${ }^{1}$ Department of Chemical and Petroleum Engineering, Schulich School of Engineering, University of Calgary \\ Calgary, AB, Canada, T2N 1N4 \\ mohammad.zargartaleb@ucalgary.ca; azaiez@ucalgary.ca
}

\section{Extended Abstract}

Nanotechnology has revolutionized several industries due to its unique features [1]. The nanoparticle specific properties make it quite different from other colloidal systems. These specific properties stem from the small size of the particles and their high surface area [2]. There have been several theoretical and experimental studies on the effects of nanoparticles on heat transfer and flow properties. Most of the studies are, however, focused on the effects of nanoparticles macroscopically and they have only reported whether the nanoparticles improve the heat transfer or if they have any effect on pressure drop [3]. There is a dearth of studies on nanoparticle-based heat transfer processes from particle point of view meaning how the particles distribute in the system and what are the effects of particle-particle and particle-wall interactions on the heat transfer processes. In this study, we have adopted the Lattice Boltzmann method to model nanofluid flow in pin-based microchannel heat sinks. The main focus has been on the particle distribution and its effects on heat transfer in a pin-based microchannel heat sink. The effects of particle-particle interactions which in some cases lead to particle agglomeration and deposition are also analysed. The effects of particle-wall interactions, which may lead to particle adsorption, are also taken into consideration. It is concluded that the particle distribution strongly affects the heat removal performance and not taking that into consideration may lead to up to $50 \%$ error in the predictions. It is also shown that particle properties play imperative roles in heat transfer and as the particle size increases, their effect on heat transfer decrease. Moreover, the surface energy of the particles is shown to play important roles in heat transfer. It is observed that particles with high surface energy are more prone to deposit and therefore unable to improve heat transfer. Hence, a characteristic curve has been developed, using the abovementioned observations, based on which one can choose the optimum particle size and surface energy to get the best efficiency out the chosen particle. The particle-wall interactions which lead to particle adsorption onto the pin surface are also studied. It is found that particle adsorption can have double-edged effects on heat transfer and can be either beneficial or detrimental depending on the functionality of the particles when they are adsorbed on the surface of the pins. Generally, it is shown that dealing with nanoparticles entails scrutinized thermodynamic and hydrodynamic analyses to ensure that the nanoparticles help improving the heat transfer in the system.

\section{References}

[1] L. E. Foster, Nanotechnology: science, innovation, and opportunity, Prentice Hall PTR, 2005.

[2] V. H. Grassian, "When size really matters: size-dependent properties and surface chemistry of metal and metal oxide nanoparticles in gas and liquid phase environments," Journal of Physical Chemistry, vol. C, no. 112, pp. 18303-18313, 2008.

[3] H. E. Ahmed, B. Salman, A. S. Kherbeet, M. Ahmed, "Optimization of thermal design of heat sinks: A review," International Journal of Heat and Mass Transfer, vol. 118, pp. 129-153, 2018. 